\title{
The Raga Guide. A Survey of 74 Hindustani Ragas
}

Nimbus Records

Philippe Bruguière

\section{(2) OpenEdition \\ Journals}

Édition électronique

URL : http://journals.openedition.org/ethnomusicologie/753

ISSN : 2235-7688

Éditeur

ADEM - Ateliers d'ethnomusicologie

Édition imprimée

Date de publication : 1 janvier 2001

Pagination : 254-256

ISBN : 2-8257-0723-6

ISSN : $1662-372 X$

Référence électronique

Philippe Bruguière, "The Raga Guide. A Survey of 74 Hindustani Ragas », Cahiers d'ethnomusicologie [En ligne], 13 | 2001, mis en ligne le 09 janvier 2012, consulté le 01 mai 2019. URL : http:// journals.openedition.org/ethnomusicologie/753

Ce document a été généré automatiquement le 1 mai 2019.

Tous droits réservés 


\title{
The Raga Guide. A Survey of 74 Hindustani Ragas
}

Nimbus Records

\author{
Philippe Bruguière
}

\section{RÉFÉRENCE}

The Raga Guide. A Survey of 74 Hindustani Ragas. Coffret de 4 CDs Nimbus Records NI 5536/9, accompagné d'un guide (Joep Bor, éd.), 196 pp. , 40 ill. coul., notes, glossaire, bibliographie.

1 Avec cette série de quatre disques publiée par Nimbus Records, il faut saluer un événement discographique qui fera date pour longtemps chez les amateurs de musique hindoustanie. Encartés dans la jaquette d'un véritable livre illustré, ce sont 74 enregistrements qui totalisent plus de cinq heures de musique et offrent avec bonheur et intelligence un ensemble représentatif de ragas extraits du répertoire contemporain. Joep Bor et ses collègues du conservatoire de Rotterdam n'ont pas ménagé leurs efforts pour nous proposer ici un formidable outil didactique, unique en son genre.

2 L'origine de ce projet, comme l'explique Bor dans sa préface, remonte à une quinzaine d'années lorsque lui-même étudiait cette musique. La difficulté inhérente à l'apprentissage d'un aussi vaste répertoire, réside autant dans les modalités d'expression formelle que dans la spécificité de l'organisation interne propre à chaque raga. Bor eut l'idée de demander à son professeur, le célèbre chanteur Dilip Chandra Vedi, de synthétiser en quelques lignes mélodiques les traits caractéristiques d'une cinquantaine de ragas couramment interprétés de nos jours. Cette heureuse initiative faisait écho à celle du musicologue Vishnu Narayan Bhatkhande qui, au début du siècle, entreprit la gigantesque tâche de recenser et collecter, auprès de nombreux musiciens, des centaines de compositions (bandish), thèmes mélodico-rythmiques qui traduisent parfaitement à 
eux seuls la nature du raga. L'œuvre de Bhatkhande fut ultérieurement publiée et diffusée à l'usage d'un enseignement institutionnalisé.

3 La tradition musicale de l'Inde du Nord est aujourd'hui largement appréciée de par le monde et ce succès est en grande partie dû aux aspects vivants et spontanés qui caractérisent l'interprétation d'un raga. Le terme raga lui-même exprime l'idée de coloration d'un état affectif et le développement inspiré d'un raga traduit bien cette notion en exerçant un réel pouvoir de fascination sur le public. Quels que soient les genres, les styles ou encore les "écoles ", le seul véritable objectif du musicien sera de créer le climat musical approprié afin qu'il évoque chez l'auditeur une réponse émotionnelle particulière. Cette conception esthétique, qui relève d'une théorie ancestrale, est néanmoins subordonnée à la mise en forme d'un ensemble de paramètres strictement musicaux. Outre l'association d'un raga à une heure particulière du jour ou de la nuit ou, dans certains cas à une saison, l'échelle musicale, l'intonation des notes, leur ornementation, leur agencement ou leur fréquence d'apparition, sont autant d'éléments constitutifs qui vont en déterminer la nature. On aura compris que tenter de définir un raga ne peut se faire en quelques mots.

4 Il serait tout aussi vain de n'y voir qu'un cadre statique livré à de strictes règles d'improvisations. Comme le souligne justement Bor, un raga est aussi une entité musicale dynamique qui n'a cessé d'évoluer, de se transformer, de disparaître et de renaître au cours des siècles. Certains ragas joués du temps de Bhatkhande ont à présent disparu, certains se sont modifiés et d'autres encore ont été créés depuis. Le Raga Guide témoigne avec une grande justesse de l'état du développement des ragas à la fin du $\mathrm{Xx}^{\mathrm{e}}$ siècle. Il y a fort à penser que dans les décennies à venir, ce guide sera regardé comme une source de documentation essentielle, au même titre que le sont aujourd'hui les compositions collectées par Bhatkhande.

Conçu comme un ouvrage destiné à être souvent consulté, le Raga Guide s'ouvre sur une brillante introduction où rien n'a été laissé au hasard. Sont d'abord exposées les règles fondamentales qui caractérisent le concept même de raga, suivies d'un bref historique sur les différents systèmes de classification dont il a fait l'objet depuis le $\mathrm{IX}^{\mathrm{e}}$ siècle ainsi que sur ses représentations poétiques et picturales. Vient ensuite la description analytique du déroulement d'un récital dans les deux genres majeurs que sont le dhrupad et le khyal et que complètent quelques explications très utiles sur les cycles rythmiques. La terminologie vernaculaire est traduite en des termes aisément compréhensibles et se retrouve dans un glossaire en fin d'ouvrage. Cette introduction se conclut avec la transcription du raga Alhaiya Bilaval, donnée à titre d'exemple. Un court texte d'accompagnement aide l'auditeur à mieux saisir l'agencement de la composition chantée, en regard des différentes techniques d'improvisation utilisées, et lui permet de reconnaître, grâce au découpage du déroulement des séquences cycliques, les divers ornements et traits spécifiques qui caractérisent ce raga.

6 Les 74 ragas choisis ont été sélectionnés parmi les plus joués de nos jours et sont interprétés par quatre éminents musiciens, autant réputés pour leur savoir que pour leur talent musical : le fluttiste Hariprasad Chaurasia et le joueur de sarod, Buddhadev Das Gupta se partagent, avec la chanteuse Shruti Sadolikar-Katkar et le chanteur Vidyadhar Vyas, le contenu des quatre disques. Les descriptions érudites de D. C. Vedi ont soustendu la mise en œuvre de cette ambitieuse entreprise, résultat d'un long et minutieux travail collectif. La durée des enregistrements, tous constitués à l'identique d'une ou deux phrases d'alap auxquelles succède une composition, varie de trois à six minutes. Chaque 
pièce exprime clairement l'image du raga tout comme le faisaient autrefois les musiciens qui gravèrent les premiers disques 78 tours.

7 Le guide s'ouvre avec le raga Abhogi et se referme sur Yaman, selon un ordre alphabétique également adopté pour la succession des plages sur les quatre $\mathrm{CD}$. Chaque raga est décrit sur une double page, celle de droite étant réservée aux transcriptions musicales. Sur la page de gauche, marquée d'un onglet indexé qui renvoie à l'enregistrement correspondant, sont d'abord résumées quelques informations concernant l'histoire du raga, son évolution et ses caractéristiques structurelles. Sont ensuite données les transcriptions de ses échelles ascendante et descendante ainsi que celle de son profil mélodique (chalan, «mouvement»), une sorte de "carte d'identité " très utile pour apprendre à reconnaître rapidement un raga. Chaque transcription est fournie simultanément dans les deux systèmes de notation, occidentale et indienne (la codification du système indien est expliquée, exemples à l'appui, dans les premières pages du guide). Seules n'ont été transcrites que les premières phrases musicales qui préludent à chaque composition. Lorsque celle-ci est chantée, son texte en écriture devanagari, accompagné d'une traduction, figure en fin de description. On regrettera cependant l'absence d'une translittération des pièces chantées qui aurait permis à l'auditeur de suivre le phrasé mélodique du texte de la composition pendant son exécution. Pour compléter cette superbe publication, ont été reproduites quarante illustrations picturales de ragas (raga-ragini) datant du XVII ${ }^{\mathrm{e}}$ siècle et dont la fonction, tout comme les versets ( dhyana) qui les accompagnent, consiste pour chacune d'entre elles à évoquer l'atmosphère du raga qu'elles représentent.

8 Avec ce Raga Guide, les aficionados de musique hindoustanie comme les étudiants ont enfin à leur disposition un ouvrage incontournable qui est déjà devenu une référence en la matière. Depuis sa parution, il y a un peu plus d'un an, le Raga Guide a rencontré un tel succès qu'il vient de faire l'objet d'un second tirage. Assurément, les nombreux dieux et déesses du panthéon hindou se sont entendus pour prêter longue vie au Raga Guide.

\section{AUTEURS}

\section{PHILIPPE BRUGUIÈRE}

fr 